\title{
FINES, VALORES Y PRINCIPIOS COMUNES A LA PROPIEDAD INTELECTUAL, AL DERECHO A LA COMPETENCIA Y A OTROS DERECHOS
}

\author{
COMMON AIMS, VALUES AND PRINCIPLES OF INTELLECTUAL PROPERTY,
} RIGHT TO COMPETENCE AND OTHERS RIGHTS

\section{Juan Carlos Riofrío Martínez-Villalba*}

Resumen: El estudio se centra en definir cuáles son los fines, valores y principios comunes a la propiedad intelectual, al derecho de la competencia, de la publicidad, del consumidor y de la información. Se muestra cómo los principios se anclan en los valores, y estos a su vez en los fines del derecho, haciéndose palpable la jerarquía que tales fines, valores y principios tienen en el ordenamiento jurídico. De esta manera, el resultado de la investigación es triple: (i) definición de los fines, valores y principios comunes a estas ramas del derecho, (ii) su interrelación; y, (iii) su jerarquización.

Palabras clave: Principios del derecho, fines del derecho, valores del derecho, veracidad, libertad

Abstract: This paper aims the definition of which are the pourposes, values and common principles of intellectual property, competence law of advertising, consumer and information. It shows how the principles are anchored in values, and these in turn into rights purposes, making palpable the hierarchy such purposes, values and principles have in the legal system. Thus, the outcome of the research is threefold: (i)

* Doctor en Jurisprudencia por la Universidad Católica Santiago de Guayaquil. Doctorando en la Pontificia Università della Santa Croce, Roma. Profesor de Derecho constitucional y de derecho de la información en la Universidad de Los Hemisferios (Quito, Ecuador). jcriofrio@coronelyperez.com 
definition of the purposes, values and principles common to these areas of law, (ii) its interface, and (iii) their ranking.

Key words: Law principles, law aims, law values, truth, freedom

Fecha de recepción: 1-12-2012

Fecha de aceptación: 21-12-2012

Fecha de publicación electrónica: 31-12-2012

Sumario. I. Fines y valores. II. Principios del derecho. III. Importancia de los principios del derecho. Referencias.

Existe un conjunto de ciencias jurídicas relacionadas de forma directa o indirecta con la información, que se hallan estrechamente interrelacionadas, sobre todo en lo que se refiere a los fines, valores y principios del Derecho. Aquí estudiamos ese núcleo común que tiene entre sí la propiedad intelectual, el derecho de la competencia (especialmente la competencia leal), el derecho de la publicidad, del consumidor y, en general, el derecho de la información.

Empezamos la exposición hablando de los fines y valores del derecho económico, para luego adentrarnos en el análisis de los principios de este derecho.

\section{FINES Y VALORES}

Para explicar el tema de los fines del Derecho - del derecho a la leal competencia, a la publicidad, al consumidor, etc.- suelo comenzar lanzando un desafío de orden existencial, que en realidad es sólo una pregunta. Entre los índices y test que miden el grado de profundidad intelectual hay uno que consiste en determinar cuántos "porqués seguidos" puede formularse una persona. Pongamos un sencillo ejemplo: ¿por qué estamos hoy y 
ahora aquí sentados leyendo este artículo? Muchas respuestas podrían darse: porque me cayó en las manos, porque necesito resolver una duda, porque me han asignado su lectura para revisarlo... Ese "para" ya nos lleva a una razón algo más profunda: debo leerlo para aprender, o quizá para obtener un bello cartón, o para subir en el escalafón... Alguien más profundo podría plantearse otro "para": ¿para qué uno quiere aprender, para qué el cartón, para qué subir en el escalafón? Si seguimos planteando porqués y para qué, veremos que esta lectura del artículo, que la profesión donde laboramos, que nuestro quehacer diario siempre tienen un fin ulterior: trabajamos para sobrevivir, para nuestra familia, para la sociedad... y aún podríamos avanzar más allá: ¿para qué sobrevivir yo y mi familia, que tantas canas me sacan? ¿Hay acaso una buena razón para no suicidarse? Muchos filósofos que se han planteado con radicalidad estas preguntas no han logrado contestarlas. Sartre llegó a afirmar que el hombre es una "pasión inútil", que vive afanándose por mil cosas que no le dan la felicidad. No deseo que ningún lector se suicide; simplemente intento mostrar que debe haber un fin último que justifique profundamente todo nuestro ser y nuestras acciones; debe haber algo último por lo que de verdad valga la pena vivir, $\mathrm{y}$ darse, y gastar todas nuestras energías. Triste vida la del que no descubre su fin último; a los 40 o 50 años su vida habrá perdido todo sentido, todo sabor.

Conviene ahora trasladar estas consideraciones existenciales al campo del derecho de la competencia. Y para hacerlo planteamos un desafío análogo al anterior. En el Ecuador, donde se acaba de expedir una nueva ley de mercado, la pregunta podría formularse en los siguientes términos: ¿por qué en Ecuador se prohíbe a los comerciantes realizar actos que confundan al consumidor? Una frecuente respuesta, quizá no muy sesuda, diría: "porque la ley lo dice". Si bien es cierto que la nueva Ley Orgánica de Regulación y Poder de Mercado, en su art. 27, num. 1, lo proscribe como supuesto específico de la competencia 
desleal, tal respuesta nos sabe a poco. Debe haber alguna otra razón. De hecho, la misma Ley en el art. 26 § 1.in fine, contiene una justificación adicional: los supuestos desleales específicos - como el anterior - están prohibidos «cuando impidan, restrinjan, falseen o distorsionen la competencia, atenten contra la eficiencia económica, o el bienestar general o los derechos de los consumidores o usuarios». En el fondo late la convicción de que la competencia, la eficiencia económica, el bienestar general y los derechos de los consumidores son un valor más último que lícitamente no se puede transgredir, y que sirven para delimitar cuándo un acto de confusión, imitación o comparación puede ser leal o desleal. El texto leído es claro, pero aún esconde varias interrogantes: la primera es si todos los valores mencionados se encuentran en el mismo nivel jerárquico o no, y la segunda es si aún dentro de esos valores cabe indagar un "por qué" o un "para qué" más último.

Considero que esta última pregunta también encuentra una satisfactoria explicación en la misma Ley, que acertadamente recoge un fundamento más profundo. En el art. 4 expresamente se dice que «en concordancia con la Constitución de la República y el ordenamiento jurídico vigente, los siguientes lineamientos se aplicarán para la regulación y formulación de política pública en la materia de esta ley: 1 . El reconocimiento del ser humano como sujeto y fin del sistema económico» ${ }^{1}$. Y esta simple frase, que

\footnotetext{
${ }^{1}$ También es buena la redacción boliviana, que dice que el Estado « tiene como máximo valor al ser humano, y asegura el desarrollo equitativo mediante la redistribución de los excedentes económicos en políticas sociales, de salud, educación y cultura» (Constitución, art. $1 \S 2$ ). Una disposición análoga consta en la Constitución peruana, que dice que «la defensa de la persona humana y el respeto de su dignidad son el fin supremo de la sociedad y del Estado» (art. 1). También consta en la Constitución venezolana que estipula que «el Estado tiene como fines esenciales la defensa y el desarrollo de la persona y el respeto a su dignidad» (art. 3). La Constitución colombiana no recoge de forma tan clara este fin último, aunque podrían encontrarse de manera dispersa dentro del articulado, especialmente en los arts. 1 y 2.
}

Ius Humani, v. 3 (2012/13), p. 40 
quizá a muchos ya les suene a pura filosofía, da precisamente en el clavo. Si la "competencia" y la "eficiencia" son un valor jurídico, lo son sólo en tanto las acciones competitivas y eficientes efectivamente estén encaminadas al beneficio humano, tanto objetiva como subjetivamente. El "bienestar general" no deja de ser un concepto etéreo si no se concreta en beneficios para todos los agentes del mercado, incluidos consumidores, productores y vendedores, que como es obvio también son persona. Como saben los versados en la materia, gran parte de los estudios del derecho de la competencia se dedican a evaluar los pros y contras que una decisión de la autoridad puede ocasionar, y esto está bien mientras las medidas políticas no pasen por encima de los derechos adquiridos de los diversos agentes del mercado.

En cuanto a la pregunta de si todos los valores jurídicos se encuentran en un mismo plano o nivel jerárquico es evidente que no. No vale lo mismo, ni merece la misma protección legal, la vida de las personas que la posibilidad de adquirir una amplia variedad de tintes para las uñas de óptima calidad. La doctrina, la ley y la Constitución ${ }^{2}$ reflejan una acertada jerarquía axiológica en este campo: primero está el valor de la persona, que debe ser tratada como fin. Spaemann decía que tal valor es tan inconmensurable, tan superior, que «esa es la razón por la que no hablamos de valor en el hombre, sino de dignidad» (Spaemann, 2000, pág. 181). Luego de la dignidad humana, en un segundo piso estarían otros fines o valores, directamente vinculados con la persona, como los derechos humanos, naturales y

${ }^{2}$ De hecho, el derecho a la vida suele ser el primer derecho reconocido en las tablas constitucionales de derechos. V. gr. Constitución peruana, art. 2, num. 1; Constitución colombiana, art. 11; Constitución venezolana, art. 43; Constitución boliviana, art. 15, inc. I. Resulta excepcional la norma ecuatoriana, que acusa una mala estructura, y luego de consagrar muchos derechos, en el art. 66 se hace una larga tabla de derechos "de libertad", donde el primero es el derecho a la vida; además, el num. 2 reconoce y garantizará «el derecho a una vida digna» (num. 2). Aun así, este derecho sigue manteniendo su peso frente a los demás (cfr. Riofrío, 2012, págs. 22-27). 
fundamentales. Dentro de todos ellos la Constitución considera fundamentales los que tienen que ver con la subsistencia humana (v. gr. la vida, el alimento, la salud) y con las más altas potencias de nuestra naturaleza: la capacidad de que el intelecto llegue a la verdad y la capacidad de la voluntad de poseer y dominar las cosas. La vida, la subsistencia, la verdad y la propiedad son valores jurídicos de altísimo rango. Por eso la Constitución suele dar un tratamiento económico especial a algunas de estas materias, prohibiendo expresamente las prácticas monopólicas en el campo alimentario ${ }^{3}$ y en los medios de comunicación ${ }^{4}$. Pero debe cuidarse no dar el mismo tratamiento a estos campos prioritarios, que requieren un cuidado especial, a otros sectores de la economía - como la compraventa de los tintes de uñasdonde no imperan razones tan urgentes.

\section{En un tercer piso aparecerían como valores jurídicos la eficiencia en el mercado, la productividad y la competencia.}

3 La Constitución ecuatoriana da mucho realce al tema alimentario, relacionándolo con la regulación del mercado. Su art. 281 dice: «La soberanía alimentaria constituye un objetivo estratégico y una obligación del Estado para garantizar que las personas, comunidades, pueblos y nacionalidades alcancen la autosuficiencia de alimentos sanos y culturalmente apropiado de forma permanente. Para ello, será responsabilidad del Estado: (...) 10. Fortalecer el desarrollo de organizaciones y redes de productores y de consumidores, así como las de comercialización y distribución de alimentos que promueva la equidad entre espacios rurales y urbano. 11. Generar sistemas justos y solidarios de distribución y comercialización de alimentos. Impedir prácticas monopólicas y cualquier tipo de especulación con productos alimenticios». Sobre ese valor concreto de la equidad, cfr. Coronel Jones, 2008, págs. 201228.

La garantía sobre los derechos alimentarios también aparece con mucha fuerza en la Constitución boliviana (cfr. arts. 16, 76, 83, 111, 254, 302, 312, 319, 373, 398, 400 y 403); algo menos en la Constitución colombiana (cfr. arts. 44 a 46, y 65), y mucho menos en la Constitución venezolana (cfr. arts. 156 y 305).

${ }^{4}$ Interesa resaltar que la Constitución ecuatoriana, en su art. 17, declara que «el Estado fomentará la pluralidad y la diversidad en la comunicación, y al efecto: (...) 3. No permitirá el oligopolio o monopolio, directo ni indirecto, de la propiedad de los medios de comunicación y del uso de las frecuencias».

Ius Humani, v. 3 (2012/13), p. 42 
Estos valores, que también constan en el texto constitucional, son desde cierto punto de vista secundarios, porque no encuentran en sí mismos su razón de ser. Producir por producir, ser eficiente por ser eficiente, competir por competir, o es un juego de mesa que sólo sirve para divertirse, o no sirve para nada. Se producen bienes para que alguien los consuma; se es eficiente para llegar a más personas; se compite libremente para ejercitar el derecho de propiedad del competidor y para facilitar al consumidor la satisfacción de sus necesidades. Sin los valores del segundo piso, los valores del tercer piso no se entienden: no hay un "por qué" que los justifique.

Este cuadro axiológico perfectamente delineado por la Constitución y por las normas reguladoras del mercado, es el que rige los principios del derecho de la competencia, de la propiedad intelectual y de otras materias afines.

\section{PRINCIPIOS DEL DERECHO}

Los principios jurídicos son directrices, postulados de razón que señalan un norte en la actuación humana. Por lo mismo, señalan hacia algo, indican un fin, una meta a conseguir, un valor a alcanzar. Y es por esto que hemos comenzado hablando de los fines y valores del derecho, que están tan relacionados, pues, según Hervada, «valor es la estimación del ser como bien, que obedece a una dimensión objetiva y real del ser» (Hervada, 2000, pág. 68), y solo se estima como bueno lo que nos ayuda a alcanzar nuestros fines. En conclusión, fines, valores y principios están absolutamente interconectados entre sí. Los fines marcan los valores, y de ambos penden los principios más generales del Derecho.

Del primer valor, el de la persona humana, se desprende el principio humano y constitucional pro homine. Este in genere contiene el resto de principios jurídicos, como el principio de igualdad, el de trato favorable al débil, etc. Por estar en lo más 
alto del escalonado orden jurídico, ellos imperan en toda rama del Derecho, y tanto también en la rama de la competencia.

En una segunda instancia encontraríamos los principios derivados de los fines y valores de segundo piso. Como dijimos, en este nivel hay dos valores fundamentales: uno que tiene que ver con la voluntad humana y otro con su inteligencia. Del primer valor se desprende el principio pro libertate, que consta por doquier en la Constitución: en la actividad privada, en el campo de la contratación, en el comercio, en las relaciones familiares, etc. ${ }^{5}$ Una concreción de este principio es el principio pro actione, que favorece la eficacia y reconoce jurídicamente efectos útiles a las acciones de las personas, tanto en el sector privado, como en el público, ante cualquier autoridad. Pero se ha de acotar que sólo se valora la voluntad buena, no la mala, dolosa o colusoria. De ahí deriva el principio de buena fe, que como bien saben mis colegas impera tanto en el derecho de la competencia, como en el derecho de marcas, como en cualquier rama del Derecho.

Mas nos interesa ahora tratar del supremo valor de la Verdad. En los tiempos que corren la sociedad se ha vuelto bastante incrédula, bastante relativista... cada uno tiene "su verdad", "su moral", "sus principios", quizá sin percatarse de lo imposible de la empresa relativista. Y aun así, la Verdad sigue siendo un valor humano y constitucional, supremo e indiscutible, máxime en el derecho de la competencia y en propiedad intelectual. Sino que pruebe un vendedor de computadores a decir "su verdad" en una publicidad comparativa donde diga que "que sus procesadores corren al doble de velocidad que los de la competencia". Si no pudiera comprobar "su verdad" —mal

5 Estos principios son de amplia aceptación, aunque algunas normas de tendencia más socialista no consagran la libertad de comercio sino de manera indirecta. En la Constitución ecuatoriana, por ejemplo, se reconoce «el derecho a desarrollar actividades económicas, en forma individual o colectiva, conforme a los principios de solidaridad, responsabilidad social y ambiental», y «el derecho a la libertad de contratación» (art. 66, nums. 15 y 16, respectivamente). Ius Humani, v. 3 (2012/13), p. 44 
llamada "suya", porque la Verdad es de todos- se reputará desleal ese acto, por contrariar:

(i) las leyes de competencia que en general sólo admiten la comparación publicitaria que se refiera a extremos análogos, relevantes y comprobables ${ }^{6}$;

(ii) la cláusula general contenidas en sendas leyes que reprimen toda competencia desleal ${ }^{7}$;

(iii) los fines de la competencia previstos en las mismas leyes ${ }^{8} ; \mathrm{y}$,

(iv) los fines, valores y principios constitucionales ya mencionados.

La Verdad es el eje sobre el cual gira gran parte de la propiedad intelectual y del derecho a la leal competencia. De ella se derivan los principios de no confusión, de no engaño, de no imitación ilícita, de distintividad de los signos (marcas, slogans, apariencias, etc. $)^{9}$ y el principio de unidad de los signos distintivos que aún no ha sido muy desarrollado en nuestro medio,

${ }^{6}$ V. gr. la Ley Orgánica de Regulación y Control de Poder de Mercado ecuatoriana, art. 27, num. 5; el Decreto Legislativo 1044 del Perú, art. 12; la Ley 256 colombiana, art. 13; y, la Ley 3/1991 española, art. 10.

7 V. gr. la Ley Orgánica de Regulación y Control de Poder de Mercado ecuatoriana, art. 26; el Decreto Legislativo 1044 del Perú, arts. 6 y 7; la Ley 256 colombiana, art. 7; y, la Ley 3/1991 española, art. 5.

8 V. gr. la Ley Orgánica de Regulación y Control de Poder de Mercado ecuatoriana, arts. 1 y 4 . Menos completos constan los fines en el art. 1 del Decreto Legislativo 1044 peruano, de la Ley 256 colombiana y de la Ley 3/1991.

${ }^{9}$ Las leyes de propiedad intelectual siempre protegen la distintividad de los signos y la imitación de las formas protegidas. A su vez, la regulación de la competencia desleal suele proscribir la confusión y el engaño, siendo generalmente los primeros supuestos prohibidos. Cfr. en Ecuador la Ley Orgánica de Regulación y Control del Poder de Mercado (2011), art. 27; en Venezuela la Ley para Promover y Proteger el Ejercicio de la Libre Competencia (1992), art. 17; en Colombia la Ley 256 (1996), arts. 10 y 11; en España la Ley 3/1991, arts. 6 y 7; en Perú el Decreto Legislativo 1044, arts. 8 y 9. La Ley 22.802 argentina de "lealtad comercial" que regula más la publicidad, que la competencia, también contiene esos dos supuestos. 
pese a estar muy desarrollado en varios países europeos ${ }^{10}$. Estos principios están recogidos en las leyes de las dos materias y son bastante conocidos por quienes se dedican ellas. Además del valor jurídico de la Verdad también se derivan otros principios que se estudian en el Derecho de la información, pero que también imperan en estas ramas del Derecho. A saber, ellos son: el principio de objetividad por el que debe transmitirse la información de la realidad tal cual es, el principio de autenticidad por el que el informante debe presentarse sinceramente como quien es, los principio de relevancia e integridad por los que deben comunicarse todos los mensajes relevantes dentro de cada contexto comunicativo, el principio de precisión por el que debe usarse un lenguaje adecuado para expresar fidedignamente lo que se informa, y el principio de honestidad que determina que en toda comunicación las partes deben actuar honestamente $\mathrm{y}$ transmitir mensajes honestos ${ }^{11}$.

Todos estos principios son comunes a tres ciencias jurídicas: al derecho de la información, al derecho de la competencia y a la propiedad intelectual. Pero cada una los aplica para sus fines propios. En concreto, al derecho de la información le interesan las transgresiones de la verdad por el simple hecho de que este valor supremo se ha transgredido. En cambio al derecho marcario no le interesa toda la verdad, pues sólo protege la verdad que permite determinar el origen o procedencia de la mercadería (por eso tanto

\footnotetext{
${ }^{10}$ Otros principios propios de la ciencia marcaria también derivan de la Verdad, como por ejemplo lo son: el principio de libre opción del signo (que aún no distinga, ni cause confusión), los principios de especialidad, temporalidad y territorialidad por los que un signo sólo puede usarse en el sector, tiempo y territorio donde efectivamente distinga, el principio de mínimo uso, el de registrabilidad y el de legítima defensa marcaria (cfr. Riofrío Martínez-Villalba, 2003a y b; Fernández-Novoa, 1984, págs. 14-119).

11 Es nuestra la presente clasificación de principios del mensaje en la comunicación. Para confrontar otras clasificaciones de principios del derecho de la información (cfr. Desantes Guanter et al., 1994, págs. 6-100; Bel Mallen et al., 1992, págs. 9-150; Terrou, 1952).
}

Ius Humani, v. 3 (2012/13), p. 46 
afán tiene en distinguir bien los signos, en evitar confusiones en las indicaciones de origen, en las apariencias distintivas, etc.). Al derecho a la leal competencia sólo le interesa el principio de veracidad en cuanto este fija un "estándar debido de lealtad" en la concurrencia; algo semejante sucede en el derecho de la publicidad, pero más con miras a los agentes de publicidad y al público general ${ }^{12}$. Por último, las leyes del consumidor precautelan la verdad para que el consumidor pueda escoger libremente los productos y servicios ofrecidos, pues sin verdad no hay libertad. Pero el principio general del derecho de veracidad es el mismo, y en todos estos campos es común que se exija un determinado nivel de verdad, y que en ocasiones se admita la antigua exceptio veritatis ante ciertas conductas informativas.

\section{IMPORTANCIA DE LOS PRINCIPIOS DEL DERECHO}

Resulta de suma importancia tener todo este cuadro axiológico y teleológico del Derecho claro. Los principios jurídicos no son meras consideraciones filosóficas destinadas a permanecer flotando en la estratósfera. Por el contrario, tienen grandes consecuencias prácticas que han sido muy estudiadas por autores de la talla de Diez-Picazo, De Castro ${ }^{13}$, Sánchez de la Torre $^{14}$ o Ferreira Rubio. Esta última autora ha sintetizado en

\footnotetext{
${ }^{12}$ Algunas normas relacionadas con la publicidad lo recogen expresamente. En este sentido, el Decreto Especial 3466 colombiano de 1982, en su art. 14, dispone que «toda información que se dé al consumidor acerca de los componentes y propiedades de los bienes y servicios que se ofrezcan al público deberá ser veraz y suficiente».

13 El autor sostiene que los principios cumplen tres funciones: a) ser fundamento del ordenamiento jurídico; b) ser orientadores de la labor de interpretación, y c) servir de fuente en caso de insuficiencia de ley o costumbre (cfr. De Castro, 1955, pág. 473).

${ }^{14}$ Para Sánchez de la Torres los principios generales del Derecho desarrollan varias misiones: 1) interpretadora; 2) integradora, 3) programática; 4) limitadora; 5) sistematizadora (cfr. Sánchez de la Torre, 1975, págs. 123-150).
} 
cuatro las funciones propias de los principios generales del derecho. Según su criterio, ellos operan: «a) como criterios informadores del total ordenamiento jurídico; b) como criterios interpretativos; c) como criterios limitativos de los derechos, y d) como criterios integradores» (Ferreira Rubio, 1984, pág. 52).

$\mathrm{Y}$ en efecto, se observa que los fines, valores y principios generales del derecho operan de estas cuatro formas frente a las cláusulas generales prohibitivas de la competencia desleal, y también frente a los supuestos legales específicos que contrarían la libre y leal competencia. Los principios sirven para leer con más profundidad la norma escrita, para interpretarla, para darle sentido, para entender la cláusula aislada de forma armónica en el ordenamiento jurídico ecuatoriano, y también sirven para limitar los abusos que eventualmente se podrían perpetrar en una mala lectura de la ley.

Con frecuencia es la jurisprudencia la que ajusta las partes defectuosas de la ley y de la regulación, que quizás ha sido redactada con una mala o descuidada técnica legislativa, o que ha olvidado regular un punto específico de derecho. Y cuando lo hace generalmente debe apelar a los fines, valores y principios más altos del ordenamiento jurídico vigente.

Una vez escuché que había algo que permitía distinguir al positivista, al burócrata, al mero técnico del Derecho, del verdadero conocedor de la materia jurídica. Cuando a ambos se les presentaba un conflicto jurídico, la pregunta inicial que se formulaba el burócrata era: ¿qué dice la ley en este caso? En cambio, el jurista se preguntará: ¿qué es lo justo aquí y ahora? Esta segunda pregunta no se podrá resolver si no tenemos claros cuáles son los fines, valores y principios más fundamentales del derecho. Sinceramente espero que todos seamos juristas y no meros técnicos del derecho. 


\section{REFERENCIAS}

Bel Mallen, I., Corredoira y Alfonso, L. \& Cousido, P. (1992). Derecho de la Información, tomo I. Madrid: Colex.

Coronel Jones, C. (2008). Los seis errores más comunes en la interpretación jurídica ecuatoriana. Ius Humani. Revista de derecho, 1, págs. 201228.

De Castro, F. (1955). Derecho Civil de España, Parte General. Madrid: Instituto de Estudios Políticos.

Desantes Guanter, J.M., Bel Mallen, I., Corredoira y Alfonso, L., Cousido, P. \& García Sanz, R.M. (1994). Derecho de la Información, tomo II. Madrid: Colex.

Fernández-Novoa, C. (1984). Fundamentos de Derecho de Marcas. Madrid: Montecorvo.

Ferreira Rubio, D. (1984). La Buena Fe. Madrid: Montecorvo.

Hervada Xiberta, J. (2000). Lecciones Propedéuticas de Filosofía del Derecho. Pamplona: Eunsa.

Riofrío Martínez-Villalba, J.C. (2003a). Principios del Derecho de Marcas. Intellectualis Dominus, 1.

Riofrío Martínez-Villalba, J.C. (2003b). Principios del Derecho de Marcas en la Comunidad Europea. Noticias jurídicas.

Riofrío Martínez-Villalba, J.C. (2012). "El Fin Último de la Constitución”. Novedades Jurídicas, 73, vol. IX, págs. 22-27.

Sánchez de la Torre, Á. (1975). Los principios clásicos del Derecho. Madrid: Unión Ed.

Spaemann, R. (2000). Personas: Acerca de la distinción entre "algo" y "alguien”, (trad. J. L. del Barco). Pamplona: Eunsa.

Terrou, F. (1952). El Derecho de la Información. París: UNESCO.

\section{Normativa citada:}

Argentina: Ley 22.802.

Bolivia: Constitución (2009) y Decreto Supremo 29519 de 16 de abril de 2008. 
Colombia: Constitución (1991) y Ley 256 (1996).

Ecuador: Constitución (2008) y Ley Orgánica de Regulación y Control de Poder de Mercado ecuatoriana (2011).

España: Ley 3/1991.

Perú: Constitución (1993) y Decreto Legislativo 1044.

Venezuela: Constitución (1999) y Ley para Promover y Proteger el Ejercicio de la Libre Competencia (1992). 